УДК 517.9

\title{
Two-layer Model of Reflective Ferromagnetic Films in Terms of Magneto-optical Ellipsometry Studies
}

\author{
Olga A. Maximova* \\ Sergey G. Ovchinnikov ${ }^{\dagger}$ \\ Kirensky Institute of Physics \\ Federal Research Center KSC SB RAS \\ Akademgorodok, 50/38, Krasnoyarsk, 660036 \\ Siberian Federal University \\ Svobodny, 79, Krasnoyarsk, 660041 \\ Russia \\ Nikolay N. Kosyrev $^{\ddagger}$ \\ Kirensky Institute of Physics \\ Federal Research Center KSC SB RAS \\ Akademgorodok, 50/38, Krasnoyarsk, 660036 \\ Sergey A. Lyaschenko ${ }^{\S}$ \\ Kirensky Institute of Physics \\ Federal Research Center KSC SB RAS \\ Akademgorodok, 50/38, Krasnoyarsk, 660036 \\ Reshetnev Siberian State Aerospace University \\ Krasnoyarsky Rabochy, 31, Krasnoyarsk, 660037
}

Russia

Received 17.11.2016, received in revised form 16.01.2017, accepted 02.02.2017

An approach to analysis of magneto-optical ellipsometry measurements is presented. A two-layer model of ferromagnetic reflective films is in focus. The obtained algorithm can be used to control optical and magneto-optical properties during films growth inside vacuum chambers.

Keywords: Magneto-optical ellipsometry, Kerr effect, two-layer model, ferromagnetic metal, reflection, growth control.

DOI: $10.17516 / 1997-1397-2017-10-2-223-232$.

Recently it has become necessary to synthesize new materials that would be applied in spintronics devices. This field of study has significantly developed and it dictates the properties that materials should have in order to be used for its purposes. It is well-known that the simplest method of generating a spin-polarised current in a metal is to pass the current through a ferromagnetic material. That is why, one of the perspective materials for spintronics is a ferromagnetic/semiconductor two-layered structure [1].

\footnotetext{
*maximo.a@mail.ru

${ }^{\dagger}$ sgo@iph.krasn.ru

${ }^{\ddagger}$ kosyrev@inbox.ru

§fenics_star@mail.ru

(C) Siberian Federal University. All rights reserved
} 
In order to synthesize them and control their properties we have to use the methods that are non-destructive, precise, easy to use, applicable for in situ investigations in the high-vacuum chambers of molecular beam epitaxy. We suggest that magneto-optical ellipsometry is a technique that reflects these requirements. Magneto-optical ellipsometry usually combines the features of conventional ellipsometry and of magneto-optical Kerr effect measurements [2-6]. Applied to the sample magnetic field changes the ellipsometric parameters, this difference can be examined and used to investigate magneto-optic properties of the sample.

In this work we give detailed explanation how to analyse magneto-ellipsometric data and obtain information on magneto-optical and optical properties of the material.

\section{General approach to magneto-ellipsometric data processing}

Our approach is based on the analysis of a well-known equation that relates the experimental ellipsometric parameters $\psi$ and $\Delta$ with complex reflection coefficients corresponding to in-plane $\left(R_{p}\right)$ and out-of-plane $\left(R_{S}\right)$ light polarizations [7-8]. Ellipsometric parameters $\psi$ and $\Delta$ can be presented as a sum of conventional parameters $\psi_{0}$ and $\Delta_{0}$ measured without external magnetic field and additional ellipsometric parameters $\delta \psi$ and $\delta \Delta$ that are the result of magnetic field application. We suggest to consider real and imaginary parts of these coefficients, so we mark them by ' and " respectively:

$$
\tan \left(\psi_{0}+\delta \psi\right) \exp \left(i\left(\Delta_{0}+\delta \Delta\right)\right)=R_{p} R_{S}^{-1}=\left(R_{p}^{\prime}-i R_{p}^{\prime \prime}\right)\left(R_{S}^{\prime}-i R_{S}^{\prime \prime}\right)^{-1}
$$

We are interested in magneto-optical properties of the sample. That is why it seems to be reasonable to present reflection coefficients as a sum of magnetic (subscript 1) and non-magnetic (subscript 0) summands [9-11]:

$$
\begin{gathered}
R_{p}=R_{p p}+R_{p S}=R_{p 0}^{\prime}+R_{p 1}^{\prime}-i\left(R_{p 0}^{\prime \prime}+R_{p 1}^{\prime \prime}\right) \\
R_{S}=R_{S S}+R_{S p}=R_{S 0}=R_{S 0}^{\prime}-i R_{S 0}^{\prime \prime}
\end{gathered}
$$

This paper focuses on the case of transverse magneto-optic Kerr effect when the magnetization is perpendicular to the plane of incidence and parallel to the surface of the sample. That is why there are no magnetic summands for s-plane polarization.

From (1-3) four equations can be obtained. Two of them correspond to non-magnetic condition:

$$
\begin{gathered}
\tan \psi_{0}=\sqrt{\frac{\left(R_{p 0}^{\prime} R_{S 0}^{\prime}+R_{S 0}^{\prime \prime} R_{p 0}^{\prime \prime}\right)^{2}+\left(R_{S 0}^{\prime \prime} R_{p 0}^{\prime}-R_{p 0}^{\prime \prime} R_{S 0}^{\prime}\right)^{2}}{R_{S 0}^{\prime 2}+R_{S 0}^{\prime 2}}}, \\
\Delta_{0}=\arctan \frac{R_{S 0}^{\prime \prime} R_{p 0}^{\prime}-R_{p 0}^{\prime \prime} R_{S 0}^{\prime}}{R_{p 0}^{\prime} R_{S 0}^{\prime}+R_{S 0}^{\prime \prime} R_{p 0}^{\prime \prime}}
\end{gathered}
$$

and two equations demonstrate the influence of an external magnetic field:

$$
\begin{gathered}
\delta \Delta=\Delta-\Delta_{0}=\arctan \frac{R_{S 0}^{\prime \prime}\left(R_{p 0}^{\prime}+R_{p 1}^{\prime}\right)-R_{S 0}^{\prime}\left(R_{p 0}^{\prime \prime}+R_{p 1}^{\prime \prime}\right)}{R_{S 0}^{\prime}\left(R_{p 0}^{\prime}+R_{p 1}^{\prime}\right)+R_{S 0}^{\prime \prime}\left(R_{p 0}^{\prime \prime}+R_{p 1}^{\prime \prime}\right)}-\Delta_{0}, \\
\delta \psi=\psi-\psi_{0}=\arctan \left(F \tan \left(\psi_{0}\right)\right)-\psi_{0},
\end{gathered}
$$


where $\mathrm{F}$ is a multiplier in

$$
\begin{gathered}
\tan \left(\psi_{0}+\delta \psi\right)=F \tan \psi_{0}= \\
=\tan \left(\psi_{0}\right) \sqrt{1+\frac{\left(R_{p 1}^{\prime 2}+R_{p 1}^{\prime \prime 2}+2\left(R_{p 0}^{\prime} R_{p 1}^{\prime}+R_{p 0}^{\prime \prime} R_{p 1}^{\prime \prime}\right)\right)\left(R_{S 0}^{\prime 2}+R_{S 0}^{\prime \prime 2}\right)}{\left(R_{p 0}^{\prime} R_{S 0}^{\prime}+R_{S 0}^{\prime \prime} R_{p 0}^{\prime \prime}\right)^{2}+\left(R_{S 0}^{\prime \prime} R_{p 0}^{\prime}-R_{p 0}^{\prime \prime} R_{S 0}^{\prime}\right)^{2}}} .
\end{gathered}
$$

These equations do not depend on the number of layers in a sample, so can be used for every type of reflective nanostrustures models. Below, a two-layer model is presented.

\section{Data processing for the case of a two-layer model}

As it was mentioned above, ferromagnetic/semiconductor two-layer structures are a subject of interest nowadays. So in this chapter let us discuss a model consisting of an upper ferromagnetic layer 1 (the refraction index $N_{1}=n_{1}-i k_{1}$ ), a middle non-magnetic layer 2 (the refraction index $N_{2}=n_{2}-i k_{2}$ ) and a substrate 3 (the refraction index $N_{3}=n_{3}-i k_{3}$ ). The light electromagnetic wave is incident from non-magnetic dielectric medium 0 (e.g. vacuum, characterized by the refraction index $N_{0}=n_{0}-i k_{0}$ ) onto the upper layer. In the setup, a Cartesian coordinate system is defined with the $\mathrm{x}$ axis normal to the interfaces and pointing into the substrate from the sample surface. The $\mathrm{y}$ and $\mathrm{x}$ axis lie in the plane of incidence. We consider T-configuration (transverse) in which magnetization is z-axis directed, i.e perpendicular to the plane of incidence and parallel to the surface. So YX plane is a plane of incidence, YZ plane is a boundary plane.

For a two-layer model it is necessary to consider each interface $(0-1,1-2,2-3)$ as each of them impacts the values of ellipsometric angles. The purpose of the data processing is to characterize a ferromagnetic layer.

The first step is carrying out ellipsometric and magneto-ellipsometric measurements. Here we do not focus on ellipsometric data analysis as there is a lot of research in this field $[7,8,12]$. So from ellipsometric measurements we can find complex refractive indices $N_{0}, N_{1}, N_{2}, N_{3}$, thicknesses of both layers, while magneto-ellipsometric parameters spectra are necessary for magnetooptical properties study of a ferromagnetic layer.

Fresnel coefficients that reflect magneto-optical properties can be derived from the scattering matrix:

$$
\hat{S}=\hat{I}_{01} \hat{L}_{1} \hat{I}_{12} \hat{L}_{2} \hat{I}_{23}
$$

where $\hat{I}_{a b}$ is an interface matrix and $\hat{L}_{c}$ is a layer matrix [7].

$$
\begin{gathered}
R_{S}=\frac{\left(S_{21}\right)_{S}}{\left(S_{11}\right)_{S}}, \\
R_{p}=\frac{\left(S_{21}\right)_{p}}{\left(S_{11}\right)_{p}}, \\
R_{S}=\frac{r_{01 S}+r_{12 S} e^{-i 2 \beta_{1}}+r_{01 S} r_{12 S} r_{23 S} e^{-i 2 \beta_{2}}+r_{23 S} e^{-i 2\left(\beta_{1}+\beta_{2}\right)}}{1+r_{01 S} r_{12 S} e^{-i 2 \beta_{1}}+r_{12 S} r_{23 S} e^{-i 2 \beta_{2}}+r_{01 S} r_{23 S} e^{-i 2\left(\beta_{1}+\beta_{2}\right)}}, \\
R_{p}=\frac{r_{01 p}+r_{12 p} \tau_{01 p} e^{-i 2 \beta_{1}}-r_{01 p} r_{21 p} r_{23 p} e^{-i 2 \beta_{2}}+r_{23 p} \tau_{01 p} \tau_{12 p} e^{-i 2\left(\beta_{1}+\beta_{2}\right)}}{1-r_{10 p} r_{12 p} e^{-i 2 \beta_{1}}-r_{21 p} r_{23 p} e^{-i 2 \beta_{2}}-r_{10 p} r_{23 p} \tau_{12 p} e^{-i 2\left(\beta_{1}+\beta_{2}\right)}},
\end{gathered}
$$

where

$$
\tau_{01 p}=t_{10 p} t_{01 p}-r_{01 p} r_{10 p}
$$




$$
\tau_{12 p}=t_{21 p} t_{12 p}-r_{12 p} r_{21 p}
$$

So, in order to process magneto-ellipsometric data the following expressions are necessary:

$$
\begin{aligned}
& r_{01 p}=\frac{N_{1} \cos \varphi_{0}-N_{0} \cos \varphi_{1}}{N_{1} \cos \varphi_{0}+N_{0} \cos \varphi_{1}}-i \frac{2 Q N_{0}^{2} \sin \varphi_{0} \cos \varphi_{0}}{\left(N_{1} \cos \varphi_{0}+N_{0} \cos \varphi_{1}\right)^{2}}, \\
& r_{12 p}=\frac{N_{2} \cos \varphi_{1}-N_{1} \cos \varphi_{2}}{N_{2} \cos \varphi_{1}+N_{1} \cos \varphi_{2}}-i \frac{2 Q N_{1}^{2} \sin \varphi_{1} \cos \varphi_{1}}{\left(N_{2} \cos \varphi_{1}+N_{1} \cos \varphi_{2}\right)^{2}}, \\
& r_{23 p}=\frac{N_{3} \cos \varphi_{2}-N_{2} \cos \varphi_{3}}{N_{3} \cos \varphi_{2}+N_{2} \cos \varphi_{3}} \\
& r_{10 p}=\frac{N_{0} \cos \varphi_{1}-N_{1} \cos \varphi_{0}}{N_{0} \cos \varphi_{1}+N_{1} \cos \varphi_{0}}+i \frac{2 Q N_{1}^{2} \sin \varphi_{1} \cos \varphi_{1}}{\left(N_{0} \cos \varphi_{1}+N_{1} \cos \varphi_{0}\right)^{2}}, \\
& r_{21 p}=\frac{N_{1} \cos \varphi_{2}-N_{2} \cos \varphi_{1}}{N_{1} \cos \varphi_{2}+N_{2} \cos \varphi_{1}}+i \frac{2 Q N_{2}^{2} \sin \varphi_{2} \cos \varphi_{2}}{\left(N_{1} \cos \varphi_{2}+N_{2} \cos \varphi_{1}\right)^{2}}, \\
& r_{01 S}=\frac{N_{0} \cos \varphi_{0}-N_{1} \cos \varphi_{1}}{N_{0} \cos \varphi_{0}+N_{1} \cos \varphi_{1}} \\
& r_{12 S}=\frac{N_{1} \cos \varphi_{1}-N_{2} \cos \varphi_{2}}{N_{1} \cos \varphi_{1}+N_{2} \cos \varphi_{2}} \\
& r_{23 S}=\frac{N_{2} \cos \varphi_{2}-N_{3} \cos \varphi_{3}}{N_{2} \cos \varphi_{2}+N_{3} \cos \varphi_{3}} \\
& t_{01 p}=\frac{2 N_{0} \cos \varphi_{0}}{N_{1} \cos \varphi_{0}+N_{0} \cos \varphi_{1}}+i \frac{2 Q N_{0}^{3} \sin \varphi_{0} \cos \varphi_{0}}{N_{1}\left(N_{1} \cos \varphi_{0}+N_{0} \cos \varphi_{1}\right)^{2}} \\
& t_{10 p}=\frac{2 N_{1} \cos \varphi_{1}}{N_{1} \cos \varphi_{0}+N_{0} \cos \varphi_{1}}-i \frac{2 Q N_{1}^{3} \sin \varphi_{1} \cos \varphi_{1}}{N_{0}\left(N_{1} \cos \varphi_{0}+N_{0} \cos \varphi_{1}\right)^{2}} \\
& t_{12 p}=\frac{2 N_{1} \cos \varphi_{1}}{N_{2} \cos \varphi_{1}+N_{1} \cos \varphi_{2}}+i \frac{2 Q N_{1}^{3} \sin \varphi_{1} \cos \varphi_{1}}{N_{2}\left(N_{2} \cos \varphi_{1}+N_{1} \cos \varphi_{2}\right)^{2}} \\
& t_{21 p}=\frac{2 N_{2} \cos \varphi_{2}}{N_{2} \cos \varphi_{1}+N_{1} \cos \varphi_{2}}-i \frac{2 Q N_{2}^{3} \sin \varphi_{2} \cos \varphi_{2}}{N_{1}\left(N_{2} \cos \varphi_{1}+N_{1} \cos \varphi_{2}\right)^{2}} \\
& \beta_{1}=\frac{2 \pi}{\lambda} N_{1} \cos \varphi_{1} d_{1}, \\
& \beta_{2}=\frac{2 \pi}{\lambda} N_{2} \cos \varphi_{2} d_{2},
\end{aligned}
$$

where $\beta_{1}$ and $\beta_{2}$ are phase thicknesses of layer 1 and layer 2 , respectively, $d_{1}$ and $d_{2}$ are thicknesses of layers 1 and 2 . Subscripts $01,12,23$ correspond to the wave propagation from medium 0 to medium 1, from 1 to 2 and from 2 to 3 respectively, while subscripts 10 and 21 correspond to the backward wave propagation. Indices $r$ are refractive indices for the mentioned above interfaces, indices $t$ are transmission coefficients. Angles $\varphi_{1}$ and $\varphi_{2}$ are related with $\varphi_{0}$ (the angle of incidence) by Snell's law. $Q$ is a magneto-optical coupling parameter that is responsible for non-diagonal elements of dielectric tensor. It means that if we know this parameter we can fully describe the dielectric permitivity, not only diagonal elements. Hereinafter we present the 
formulae necessary for identifying $Q$ from magneto-ellipsometric measurements. Let us rewrite $(12-18)$ in the same manner as $(2,3)$ :

$$
\begin{gathered}
r_{01 S}=\left(R_{S 0}^{\prime}\right)_{01}-i\left(R_{S 0}^{\prime \prime}\right)_{01}, \\
r_{12 S}=\left(R_{S 0}^{\prime}\right)_{12}-i\left(R_{S 0}^{\prime \prime}\right)_{12}, \\
r_{23 S}=\left(R_{S 0}^{\prime}\right)_{23}-i\left(R_{S 0}^{\prime \prime}\right)_{23}, \\
r_{23 p}=\left(R_{p 0}^{\prime}\right)_{23}-i\left(R_{p 0}^{\prime \prime}\right)_{23}=r r_{23}-i r i_{23}, \\
r_{01 p}=\left(R_{p 0}^{\prime}\right)_{01}+\left(R_{p 1}^{\prime}\right)_{01}-i\left(\left(R_{p 0}^{\prime \prime}\right)_{01}+\left(R_{p 1}^{\prime \prime}\right)_{01}\right)=r r_{01}-i r i_{01}, \\
r_{12 p}=\left(R_{p 0}^{\prime}\right)_{12}+\left(R_{p 1}^{\prime}\right)_{12}-i\left(\left(R_{p 0}^{\prime \prime}\right)_{12}+\left(R_{p 1}^{\prime \prime}\right)_{12}\right)=r r_{12}-i r i_{12}, \\
r_{23 p}=\left(R_{p 0}^{\prime}\right)_{23}+\left(R_{p 1}^{\prime}\right)_{23}-i\left(\left(R_{p 0}^{\prime \prime}\right)_{23}+\left(R_{p 1}^{\prime \prime}\right)_{23}\right)=r r_{23}-i r i_{23}, \\
r_{10 p}=\left(R_{p 0}^{\prime}\right)_{10}-\left(R_{p 1}^{\prime}\right)_{10}-i\left(\left(R_{p 0}^{\prime \prime}\right)_{10}-\left(R_{p 1}^{\prime \prime}\right)_{10}\right)=r r_{10}-i r i_{10}, \\
r_{21 p}=\left(R_{p 0}^{\prime}\right)_{21}-\left(R_{p 1}^{\prime}\right)_{21}-i\left(\left(R_{p 0}^{\prime \prime}\right)_{21}-\left(R_{p 1}^{\prime \prime}\right)_{21}\right)=r r_{21}-i r i_{21}, \\
t_{01 p}=\left(T_{p 0}^{\prime}\right)_{01}+\left(T_{p 1}^{\prime}\right)_{01}-i\left(\left(T_{p 0}^{\prime \prime}\right)_{01}+\left(T_{p 1}^{\prime \prime}\right)_{01}\right)=t r_{01}-i t i_{01}, \\
t_{12 p}=\left(T_{p 0}^{\prime}\right)_{12}+\left(T_{p 1}^{\prime}\right)_{12}-i\left(\left(T_{p 0}^{\prime \prime}\right)_{12}+\left(T_{p 1}^{\prime \prime}\right)_{12}\right)=t r_{12}-i t i_{12}, \\
t_{10 p}=\left(T_{p 0}^{\prime}\right)_{10}-\left(T_{p 1}^{\prime}\right)_{10}-i\left(\left(T_{p 0}^{\prime \prime}\right)_{10}-\left(T_{p 1}^{\prime \prime}\right)_{10}\right)=t r_{10}-i t i_{10}, \\
t_{21 p}=\left(T_{p 0}^{\prime}\right)_{21}-\left(T_{p 1}^{\prime}\right)_{21}-i\left(\left(T_{p 0}^{\prime \prime}\right)_{21}-\left(T_{p 1}^{\prime \prime}\right)_{21}\right)=t r_{21}-i t i_{21},
\end{gathered}
$$

where $\left(R_{s 0}^{\prime}\right)_{01},\left(R_{s 0}^{\prime \prime}\right)_{01},\left(R_{p 0}^{\prime}\right)_{01},\left(R_{p 0}^{\prime \prime}\right)_{01},\left(R_{p 1}^{\prime}\right)_{01},\left(R_{p 1}^{\prime \prime}\right)_{01}$ correspond to $R_{s 0}^{\prime}, R_{s 0}^{\prime \prime}, R_{p 0}^{\prime}, R_{p 0}^{\prime \prime}, R_{p 1}^{\prime}$, $R_{p 1}^{\prime \prime}$ in the model of a homogeneous semi-infinite medium, respectively [11]. Subscript 01 denotes the electromagnetic wave incidence from ambient medium 0 onto layer 1 . Indices $\left(R_{s 0}^{\prime}\right)_{12},\left(R_{s 0}^{\prime \prime}\right)_{12},\left(R_{p 0}^{\prime}\right)_{12},\left(R_{p 0}^{\prime \prime}\right)_{12},\left(R_{p 1}^{\prime}\right)_{12},\left(R_{p 1}^{\prime \prime}\right)_{12}$ are also calculated by formulae for the model of a homogeneous semi-infinite medium, the only difference is that subscript 12 denotes the electromagnetic wave incidence from layer 1 onto layer 2 that leads to the following changes in the formulae for the model of a homogeneous semi-infinite medium: $\cos \varphi_{0} \rightarrow \cos \varphi_{1}$, $\cos \varphi_{1} \rightarrow \cos \varphi_{2}, \sin \varphi_{0} \rightarrow \sin \varphi_{1}, n_{1} \rightarrow n_{2}, n_{0} \rightarrow n_{1}, k_{1} \rightarrow k_{2}, k_{0} \rightarrow k_{1}$. Likewise, indices $\left(R_{p 0}^{\prime}\right)_{10},\left(R_{p 0}^{\prime \prime}\right)_{10},\left(R_{p 1}^{\prime}\right)_{10},\left(R_{p 1}^{\prime \prime}\right)_{10}$ describe the electromagnetic wave propagation from layer 1 to medium $0: \cos \varphi_{0} \leftrightarrow \cos \varphi_{1}, \sin \varphi_{0} \leftrightarrow \sin \varphi_{1}, n_{0} \leftrightarrow n_{1}, k_{0} \leftrightarrow k_{1}$. Indices $\left(R_{p 0}^{\prime}\right)_{21},\left(R_{p 0}^{\prime \prime}\right)_{21},\left(R_{p 1}^{\prime}\right)_{21},\left(R_{p 1}^{\prime \prime}\right)_{21}$ correspond to the electromagnetic wave propagation from layer 2 to layer 1: $\cos \varphi_{0} \rightarrow \cos \varphi_{2}, \sin \varphi_{0} \rightarrow \sin \varphi_{2}, n_{0} \rightarrow n_{2}, k_{0} \rightarrow k_{2}$. Finally, indices $\left(R_{s 0}^{\prime}\right)_{23},\left(R_{s 0}^{\prime \prime}\right)_{23},\left(R_{p 0}^{\prime}\right)_{23},\left(R_{p 0}^{\prime \prime}\right)_{23},\left(R_{p 1}^{\prime}\right)_{23},\left(R_{p 1}^{\prime \prime}\right)_{23}$ describe the electromagnetic wave incidence from layer 2 on substrate 3: $\cos \varphi_{0} \rightarrow \cos \varphi_{2}, \cos \varphi_{1} \rightarrow \cos \varphi_{3}, \sin \varphi_{0} \rightarrow \sin \varphi_{2}, n_{1} \rightarrow n_{3}$, $n_{0} \rightarrow n_{2}, k_{1} \rightarrow k_{3}, k_{0} \rightarrow k_{2}$.

Transmission coefficients necessary for data processing are the following:

$$
\begin{gathered}
\left(T_{p 0}^{\prime}\right)_{01}=2 \frac{\left(n_{0} n_{1}+k_{0} k_{1}\right)\left(a^{2}+c^{2}\right)+\left(n_{0}^{2}+k_{0}^{2}\right)(a b+c d)}{A_{3}^{2}+B_{3}^{2}}, \\
\left(T_{p 0}^{\prime \prime}\right)_{01}=2 \frac{\left(n_{0}^{2}+k_{0}^{2}\right)(a d-b c)+\left(n_{1} k_{0}-n_{0} k_{1}\right)\left(a^{2}+c^{2}\right)}{A_{3}^{2}+B_{3}^{2}}, \\
\left(T_{p 1}^{\prime}\right)_{01}=2 \frac{Q_{1}(p q+r s)-Q_{2}(p r-s q)}{\left(n_{1}^{2}+k_{1}^{2}\right)\left(A_{3}^{2}+B_{3}^{2}\right)^{2}}, \\
\left(T_{p 1}^{\prime \prime}\right)_{01}=2 \frac{Q_{1}(p r-s q)+Q_{2}(p q+r s)}{\left(n_{1}^{2}+k_{1}^{2}\right)\left(A_{3}^{2}+B_{3}^{2}\right)^{2}},
\end{gathered}
$$


where

$$
\begin{gathered}
A_{3}=n_{1} a+k_{1} c+n_{0} b+k_{0} d, \\
B_{3}=k_{1} a-n_{1} c+k_{0} b-n_{0} d, \\
p=N\left(3 n_{0}^{2} k_{0}-k_{0}^{3}\right)+P\left(n_{0}^{3}-3 n_{0} k_{0}^{2}\right), \\
q=n_{1}\left(A_{3}^{2}-B_{3}^{2}\right)-2 A_{3} B_{3} k_{1}, \\
r=k_{1}\left(B_{3}^{2}-A_{3}^{2}\right)-2 A_{3} B_{3} n_{1}, \\
s=N\left(n_{0}^{3}-3 n_{0} k_{0}^{2}\right)-P\left(3 n_{0}^{2} k_{0}-k_{0}^{3}\right), \\
a=\operatorname{Re}\left(\cos \varphi_{0}\right), \\
b=\operatorname{Re}\left(\cos \varphi_{1}\right), \\
c=\operatorname{Im}\left(\cos \varphi_{0}\right), \\
d=\operatorname{Im}\left(\cos \varphi_{1}\right), \\
N=\operatorname{Re}\left(\sin \varphi_{0}\right) a-\operatorname{Im}\left(\sin \varphi_{0}\right) c, \\
P=-\operatorname{Re}\left(\sin \varphi_{0}\right) c-\operatorname{Im}\left(\sin \varphi_{0}\right) a .
\end{gathered}
$$

Transmission coefficients with subscripts 10, 12, 21 correspond to the electromagnetic wave propagation from layer 1 to medium 0 , from layer 1 to layer 2 , from layer 2 to layer 1 , respectively. The changes in the formulae are the same as proposed for refractive indices.

Let us take into account $N_{0}=n_{0}-i k_{0}, N_{1}=n_{1}-i k_{1}, N_{2}=n_{2}-i k_{2}, Q=Q_{1}-i Q_{2}$ and compare expressions $(12,13)$ with $(2,3)$. Thus we obtain expressions for $R_{p 0}^{\prime}, R_{p 0}^{\prime \prime}, R_{p 1}^{\prime}, R_{p 1}^{\prime \prime}, R_{s 0}^{\prime}$ and $R_{s 0}^{\prime \prime}$ in terms of numerators and denominators:

$$
\begin{gathered}
R_{S 0}=\frac{\text { numerator } R_{S 0}}{\text { denominator } R_{S 0}} \equiv \frac{\operatorname{Re}\left(n\left(R_{S 0}\right)\right)-i \operatorname{Im}\left(n\left(R_{S 0}\right)\right)}{\operatorname{Re}\left(d\left(R_{S 0}\right)\right)-i \operatorname{Im}\left(d\left(R_{S 0}\right)\right)}, \\
R_{p 0}=\frac{\operatorname{Re}\left(n\left(R_{p 0}\right)\right)-i \operatorname{Im}\left(n\left(R_{p 0}\right)\right)}{\operatorname{Re}\left(d\left(R_{p 0}\right)\right)-i \operatorname{Im}\left(d\left(R_{p 0}\right)\right)}, \\
R_{p}=\frac{\operatorname{Re}\left(n\left(R_{p}\right)\right)-i \operatorname{Im}\left(n\left(R_{p}\right)\right)}{\operatorname{Re}\left(d\left(R_{p}\right)\right)-i \operatorname{Im}\left(d\left(R_{p}\right)\right)},
\end{gathered}
$$

where $n$ stands for numerator and $d$ - for denominator. As a result, we have

$$
\begin{aligned}
R_{p 0}^{\prime} & =\frac{\operatorname{Re}\left(n\left(R_{p 0}\right)\right) \operatorname{Re}\left(d\left(R_{p 0}\right)\right)+\operatorname{Im}\left(n\left(R_{p 0}\right)\right) \operatorname{Im}\left(d\left(R_{p 0}\right)\right)}{\left(\operatorname{Re}\left(d\left(R_{p 0}\right)\right)\right)^{2}+\left(\operatorname{Im}\left(d\left(R_{p 0}\right)\right)\right)^{2}}, \\
R_{p 0}^{\prime \prime} & =\frac{\operatorname{Im}\left(n\left(R_{p 0}\right)\right) \operatorname{Re}\left(d\left(R_{p 0}\right)\right)-\operatorname{Im}\left(d\left(R_{p 0}\right)\right) \operatorname{Re}\left(n\left(R_{p 0}\right)\right)}{\left(\operatorname{Re}\left(d\left(R_{p 0}\right)\right)\right)^{2}+\left(\operatorname{Im}\left(d\left(R_{p 0}\right)\right)\right)^{2}}, \\
R_{p 1}^{\prime} & =\frac{\operatorname{Re}\left(n\left(R_{p}\right)\right) \operatorname{Re}\left(d\left(R_{p}\right)\right)+\operatorname{Im}\left(n\left(R_{p}\right)\right) \operatorname{Im}\left(d\left(R_{p}\right)\right)}{\left(\operatorname{Re}\left(d\left(R_{p}\right)\right)\right)^{2}+\left(\operatorname{Im}\left(d\left(R_{p}\right)\right)\right)^{2}}-R_{p 0}^{\prime}, \\
R_{p 1}^{\prime \prime} & =\frac{\operatorname{Im}\left(n\left(R_{p}\right)\right) \operatorname{Re}\left(d\left(R_{p}\right)\right)-\operatorname{Im}\left(d\left(R_{p}\right)\right) \operatorname{Re}\left(n\left(R_{p}\right)\right)}{\left(\operatorname{Re}\left(d\left(R_{p}\right)\right)\right)^{2}+\left(\operatorname{Im}\left(d\left(R_{p}\right)\right)\right)^{2}}-R_{p 0}^{\prime \prime}, \\
R_{S 0}^{\prime} & =\frac{\operatorname{Re}\left(n\left(R_{S 0}\right)\right) \operatorname{Re}\left(d\left(R_{S 0}\right)\right)+\operatorname{Im}\left(n\left(R_{S 0}\right)\right) \operatorname{Im}\left(d\left(R_{S 0}\right)\right)}{\left(\operatorname{Re}\left(d\left(R_{S 0}\right)\right)\right)^{2}+\left(\operatorname{Im}\left(d\left(R_{S 0}\right)\right)\right)^{2}},
\end{aligned}
$$




$$
R_{S 0}^{\prime \prime}=\frac{\operatorname{Im}\left(n\left(R_{S 0}\right)\right) \operatorname{Re}\left(d\left(R_{S 0}\right)\right)-\operatorname{Im}\left(d\left(R_{S 0}\right)\right) \operatorname{Re}\left(n\left(R_{S 0}\right)\right)}{\left(\operatorname{Re}\left(d\left(R_{S 0}\right)\right)\right)^{2}+\left(\operatorname{Im}\left(d\left(R_{S 0}\right)\right)\right)^{2}},
$$

where the following notations are used:

$$
\begin{gathered}
\operatorname{Re}\left(n\left(R_{p 0}\right)\right)=\left(R_{p 0}^{\prime}\right)_{01}+\xi_{1}\left(R_{p 0}^{\prime}\right)_{12}-\eta_{1}\left(R_{p 0}^{\prime \prime}\right)_{12}+L_{0112}\left(\xi_{2}\left(R_{p 0}^{\prime}\right)_{23}-\eta_{2}\left(R_{p 0}^{\prime \prime}\right)_{23}\right)- \\
-M_{0112}\left(\xi_{2}\left(R_{p 0}^{\prime \prime}\right)_{23}+\eta_{2}\left(R_{p 0}^{\prime}\right)_{23}\right)+\left(R_{p 0}^{\prime}\right)_{23}\left(\xi_{1} \xi_{2}-\eta_{1} \eta_{2}\right)-\left(R_{p 0}^{\prime \prime}\right)_{23}\left(\xi_{2} \eta_{1}+\xi_{1} \eta_{2}\right) \\
\operatorname{Im}\left(n\left(R_{p 0}\right)\right)=\left(R_{p 0}^{\prime \prime}\right)_{01}+\eta_{1}\left(R_{p 0}^{\prime}\right)_{12}+\xi_{1}\left(R_{p 0}^{\prime \prime}\right)_{12}+L_{0112}\left(\xi_{2}\left(R_{p 0}^{\prime \prime}\right)_{23}+\eta_{2}\left(R_{p 0}^{\prime}\right)_{23}\right)+ \\
+M_{0112}\left(\xi_{2}\left(R_{p 0}^{\prime}\right)_{23}-\eta_{2}\left(R_{p 0}^{\prime \prime}\right)_{23}\right)+\left(R_{p 0}^{\prime \prime}\right)_{23}\left(\xi_{1} \xi_{2}-\eta_{1} \eta_{2}\right)+\left(R_{p 0}^{\prime}\right)_{23}\left(\xi_{2} \eta_{1}+\xi_{1} \eta_{2}\right) \\
\operatorname{Re}\left(d\left(R_{p 0}\right)\right)=1+L_{0112} \xi_{1}-M_{0112} \eta_{1}+\xi_{2} L_{1223}-\eta_{2} M_{1223}+ \\
+\left(\xi_{1} \xi_{2}-\eta_{1} \eta_{2}\right) L_{0123}-\left(\xi_{2} \eta_{1}+\xi_{1} \eta_{2}\right) M_{0123}, \\
\operatorname{Im}\left(d\left(R_{p 0}\right)\right)=L_{0112} \eta_{1}+M_{0112} \xi_{1}+\xi_{2} M_{1223}+\eta_{2} L_{1223}+ \\
+\left(\xi_{1} \xi_{2}-\eta_{1} \eta_{2}\right) M_{0123}+\left(\xi_{2} \eta_{1}+\xi_{1} \eta_{2}\right) L_{0123},
\end{gathered}
$$

$\operatorname{Re}\left(n\left(R_{p}\right)\right)=r r_{01}+\left(\xi_{1} r r_{12}-\eta_{1} r i_{12}\right)\left(\kappa_{1}\right)_{01}-\left(\xi_{1} r i_{12}+\eta_{1} r r_{12}\right)\left(\kappa_{2}\right)_{01}-$ $-\left(r r_{01} r r_{21}-r i_{01} r i_{21}\right)\left(\xi_{2} r r_{23}-\eta_{2} r i_{23}\right)+\left(r i_{01} r r_{21}+r r_{01} r i_{21}\right)\left(\xi_{2} r i_{23}+\eta_{2} r r_{23}\right)+$$$
+\left(r r_{23}\left(\xi_{1} \xi_{2}-\eta_{1} \eta_{2}\right)-r i_{23}\left(\xi_{2} \eta_{1}+\xi_{1} \eta_{2}\right)\right)\left(\left(\kappa_{1}\right)_{01}\left(\kappa_{1}\right)_{12}-\left(\kappa_{2}\right)_{01}\left(\kappa_{2}\right)_{12}\right)-
$$$$
-\left(r i_{23}\left(\xi_{1} \xi_{2}-\eta_{1} \eta_{2}\right)+r r_{23}\left(\xi_{1} \eta_{2}+\eta_{1} \xi_{2}\right)\right)\left(\left(\kappa_{1}\right)_{01}\left(\kappa_{2}\right)_{12}+\left(\kappa_{1}\right)_{12}\left(\kappa_{2}\right)_{01}\right),
$$$$
\operatorname{Im}\left(n\left(R_{p}\right)\right)=r i_{01}+\left(\xi_{1} r i_{12}+\eta_{1} r r_{12}\right)\left(\kappa_{1}\right)_{01}+\left(\xi_{1} r r_{12}-\eta_{1} r i_{12}\right)\left(\kappa_{2}\right)_{01}-
$$$$
-\left(r i_{01} r r_{21}+r r_{01} r i_{21}\right)\left(\xi_{2} r r_{23}-\eta_{2} r i_{23}\right)-\left(r r_{01} r r_{21}-r i_{01} r i_{21}\right)\left(\xi_{2} r i_{23}+\eta_{2} r r_{23}\right)+
$$$$
+\left(r i_{23}\left(\xi_{1} \xi_{2}-\eta_{1} \eta_{2}\right)+r r_{23}\left(\xi_{2} \eta_{1}+\xi_{1} \eta_{2}\right)\right)\left(\left(\kappa_{1}\right)_{01}\left(\kappa_{1}\right)_{12}-\left(\kappa_{2}\right)_{01}\left(\kappa_{2}\right)_{12}\right)+
$$$$
+\left(r r_{23}\left(\xi_{1} \xi_{2}-\eta_{1} \eta_{2}\right)-r i_{23}\left(\xi_{1} \eta_{2}+\eta_{1} \xi_{2}\right)\right)\left(\left(\kappa_{1}\right)_{01}\left(\kappa_{2}\right)_{12}+\left(\kappa_{1}\right)_{12}\left(\kappa_{2}\right)_{01}\right)
$$$$
\operatorname{Re}\left(d\left(R_{p}\right)\right)=1-\xi_{1}\left(r r_{10} r r_{12}-r i_{10} r i_{12}\right)+\eta_{1}\left(r i_{10} r r_{12}+r r_{10} r i_{12}\right)-
$$$$
-\xi_{2}\left(r r_{21} r r_{23}-r i_{21} r i_{23}\right)+\eta_{2}\left(r i_{21} r r_{23}+r i_{23} r r_{21}\right)-
$$$$
\left(\left(\kappa_{1}\right)_{12}\left(r r_{10} r r_{23}-r i_{10} r i_{23}\right)-\left(\kappa_{2}\right)_{12}\left(r i_{10} r r_{23}+r i_{23} r r_{10}\right)\right)\left(\xi_{1} \xi_{2}-\eta_{1} \eta_{2}\right)+
$$$$
\left.+\left(\left(\kappa_{1}\right)_{12}\right)\left(r i_{10} r r_{23}+r i_{23} r r_{10}\right)+\left(\kappa_{2}\right)_{12}\left(r r_{10} r r_{23}-r i_{10} r i_{23}\right)\right)\left(\xi_{2} \eta_{1}+\xi_{1} \eta_{2}\right),
$$$$
\operatorname{Im}\left(d\left(R_{p}\right)\right)=-\xi_{1}\left(r i_{10} r r_{12}+r r_{10} r i_{12}\right)-\eta_{1}\left(r r_{10} r r_{12}-r i_{10} r i_{12}\right)-
$$$$
-\xi_{2}\left(r i_{21} r r_{23}+r r_{21} r i_{23}\right)-\eta_{2}\left(r r_{21} r r_{23}-r i_{23} r i_{21}\right)-
$$$$
\left(\left(\kappa_{1}\right)_{12}\left(r i_{10} r r_{23}+r r_{10} r i_{23}\right)+\left(\kappa_{2}\right)_{12}\left(r r_{10} r r_{23}-r i_{23} r i_{10}\right)\right)\left(\xi_{1} \xi_{2}-\eta_{1} \eta_{2}\right)-
$$$$
\left.-\left(\left(\kappa_{1}\right)_{12}\right)\left(r r_{10} r r_{23}-r i_{23} r i_{10}\right)-\left(\kappa_{2}\right)_{12}\left(r i_{10} r r_{23}+r r_{10} r i_{23}\right)\right)\left(\xi_{2} \eta_{1}+\xi_{1} \eta_{2}\right) \text {, }
$$

$\operatorname{Re}\left(n\left(R_{S 0}\right)\right)=\left(R_{S 0}^{\prime}\right)_{01}+\xi_{1}\left(R_{S 0}^{\prime}\right)_{12}-\eta_{1}\left(R_{S 0}^{\prime \prime}\right)_{12}+H_{0112}\left(\xi_{2}\left(R_{S 0}^{\prime}\right)_{23}-\eta_{2}\left(R_{S 0}^{\prime \prime}\right)_{23}\right)-$

$$
-J_{0112}\left(\xi_{2}\left(R_{S 0}^{\prime \prime}\right)_{23}+\eta_{2}\left(R_{S 0}^{\prime}\right)_{23}\right)+\left(R_{S 0}^{\prime}\right)_{23}\left(\xi_{1} \xi_{2}-\eta_{1} \eta_{2}\right)-\left(R_{S 0}^{\prime \prime}\right)_{23}\left(\xi_{2} \eta_{1}+\xi_{1} \eta_{2}\right),
$$




$$
\begin{aligned}
& \operatorname{Im}\left(n\left(R_{S 0}\right)\right)=\left(R_{S 0}^{\prime \prime}\right)_{01}+\eta_{1}\left(R_{S 0}^{\prime}\right)_{12}+\xi_{1}\left(R_{S 0}^{\prime \prime}\right)_{12}+H_{0112}\left(\xi_{2}\left(R_{S 0}^{\prime \prime}\right)_{23}+\eta_{2}\left(R_{S 0}^{\prime}\right)_{23}\right)+ \\
& +J_{0112}\left(\xi_{2}\left(R_{S 0}^{\prime}\right)_{23}-\eta_{2}\left(R_{S 0}^{\prime \prime}\right)_{23}\right)+\left(R_{S 0}^{\prime \prime}\right)_{23}\left(\xi_{1} \xi_{2}-\eta_{1} \eta_{2}\right)+\left(R_{S 0}^{\prime}\right)_{23}\left(\xi_{2} \eta_{1}+\xi_{1} \eta_{2}\right), \\
& \operatorname{Re}\left(d\left(R_{S 0}\right)\right)=1+H_{0112} \xi_{1}-J_{0112} \eta_{1}+\xi_{2} H_{1223}-\eta_{2} J_{1223}+ \\
& +\left(\xi_{1} \xi_{2}-\eta_{1} \eta_{2}\right) H_{0123}-\left(\xi_{2} \eta_{1}+\xi_{1} \eta_{2}\right) J_{0123}, \\
& \operatorname{Im}\left(d\left(R_{S 0}\right)\right)=H_{0112} \eta_{1}+J_{0112} \xi_{1}+\xi_{2} J_{1223}+\eta_{2} H_{1223}+ \\
& +\left(\xi_{1} \xi_{2}-\eta_{1} \eta_{2}\right) J_{0123}+\left(\xi_{2} \eta_{1}+\xi_{1} \eta_{2}\right) H_{0123} \\
& \xi_{1}=\operatorname{Re}\left(e^{-i 2 \beta_{1}}\right), \\
& \eta_{1}=-\operatorname{Im}\left(e^{-i 2 \beta_{1}}\right) \text {, } \\
& \xi_{2}=\operatorname{Re}\left(e^{-i 2 \beta_{2}}\right) \text {, } \\
& \eta_{2}=-\operatorname{Im}\left(e^{-i 2 \beta_{2}}\right), \\
& L_{0112}=\left(R_{p 0}^{\prime}\right)_{12}\left(R_{p 0}^{\prime}\right)_{01}-\left(R_{p 0}^{\prime \prime}\right)_{12}\left(R_{p 0}^{\prime \prime}\right)_{01} \\
& M_{0112}=\left(R_{p 0}^{\prime}\right)_{01}\left(R_{p 0}^{\prime \prime}\right)_{12}+\left(R_{p 0}^{\prime \prime}\right)_{01}\left(R_{p 0}^{\prime}\right)_{12}, \\
& L_{1223}=\left(R_{p 0}^{\prime}\right)_{23}\left(R_{p 0}^{\prime}\right)_{12}-\left(R_{p 0}^{\prime \prime}\right)_{23}\left(R_{p 0}^{\prime \prime}\right)_{12} \\
& M_{1223}=\left(R_{p 0}^{\prime}\right)_{12}\left(R_{p 0}^{\prime \prime}\right)_{23}+\left(R_{p 0}^{\prime \prime}\right)_{12}\left(R_{p 0}^{\prime}\right)_{23}, \\
& L_{0123}=\left(R_{p 0}^{\prime}\right)_{23}\left(R_{p 0}^{\prime}\right)_{01}-\left(R_{p 0}^{\prime \prime}\right)_{23}\left(R_{p 0}^{\prime \prime}\right)_{01}, \\
& M_{0123}=\left(R_{p 0}^{\prime}\right)_{01}\left(R_{p 0}^{\prime \prime}\right)_{23}+\left(R_{p 0}^{\prime \prime}\right)_{01}\left(R_{p 0}^{\prime}\right)_{23}, \\
& H_{0112}=\left(R_{S 0}^{\prime}\right)_{12}\left(R_{S 0}^{\prime}\right)_{01}-\left(R_{S 0}^{\prime \prime}\right)_{12}\left(R_{S 0}^{\prime \prime}\right)_{01} \text {, } \\
& J_{0112}=\left(R_{S 0}^{\prime}\right)_{01}\left(R_{S 0}^{\prime \prime}\right)_{12}+\left(R_{S 0}^{\prime \prime}\right)_{01}\left(R_{S 0}^{\prime}\right)_{12}, \\
& H_{1223}=\left(R_{S 0}^{\prime}\right)_{23}\left(R_{S 0}^{\prime}\right)_{12}-\left(R_{S 0}^{\prime \prime}\right)_{23}\left(R_{S 0}^{\prime \prime}\right)_{12}, \\
& J_{1223}=\left(R_{S 0}^{\prime}\right)_{12}\left(R_{S 0}^{\prime \prime}\right)_{23}+\left(R_{S 0}^{\prime \prime}\right)_{12}\left(R_{S 0}^{\prime}\right)_{23}, \\
& H_{0123}=\left(R_{S 0}^{\prime}\right)_{23}\left(R_{S 0}^{\prime}\right)_{01}-\left(R_{S 0}^{\prime \prime}\right)_{23}\left(R_{S 0}^{\prime \prime}\right)_{01}, \\
& J_{0123}=\left(R_{S 0}^{\prime}\right)_{01}\left(R_{S 0}^{\prime \prime}\right)_{23}+\left(R_{S 0}^{\prime \prime}\right)_{01}\left(R_{S 0}^{\prime}\right)_{23}, \\
& \left(\kappa_{1}\right)_{01}=t r_{10} t r_{01}-t i_{10} t i_{01}-r r_{01} r r_{10}+r i_{01} r i_{10}, \\
& \left(\kappa_{2}\right)_{01}=t i_{10} t r_{01}+t r_{10} t i_{01}-r r_{01} r i_{10}-r i_{01} r r_{10}, \\
& \left(\kappa_{1}\right)_{12}=t r_{21} t r_{12}-t i_{21} t i_{12}-r r_{21} r r_{12}+r i_{21} r i_{12}, \\
& \left(\kappa_{2}\right)_{12}=t i_{12} t r_{21}+t r_{12} t i_{21}-r r_{12} r i_{21}-r i_{12} r r_{21} \text {. }
\end{aligned}
$$

So all necessary expressions that relate measured ellipsometric and magneto-ellipsometric parameters with refraction indices, coefficients of extinction, magneto-optical coupling parameter in case of a two-layer model are obtained. The final step is giving the best fit to the experimental data by the use of the wavelength-to-wavelength Nelder-Mead minimization [13] of the 
ellipsometric angles. It yields real and imaginary parts of magneto-optical parameter $Q$, thus information about all elements of the dielectric permittivity tensor can be obtained from the experiment.

\section{Conclusion}

To conclude, we have proposed an approach to studying two-layer nanomaterials by means of magneto-ellipsometry. The algorithm of experimental data analysis $\left(\psi_{0}, \delta_{0}, \psi_{0}+\delta \psi, \Delta_{0}+\delta \Delta\right)$ is presented. As a result, optical and magneto-optical properties can be easily and reliably characterized during films growth through the presented formulae that are to be used in the software for magneto-optical ellipsometry set-ups.

The reported study was funded by Russian Foundation for Basic Research, Government of Krasnoyarsk Territory, Krasnoyarsk Region Science and Technology Support Fund to the research project 16-42-243058. The work was supported partly by the Russian Foundation for Basic Research, Grant No. 16-32-00209 mol, Grant No. 14-02-01211; the Complex program of SB RAS No. II.2P, project 0358-2015-0004; the Ministry of Education and Science of the RF (State task No. 16.663.2014); grant Scientific School 7559.2016.2.

\section{References}

[1] N.V.Volkov et al., Magnetic-field- and bias-sensitive conductivity of a hybrid $\mathrm{Fe} / \mathrm{SiO}_{2} / \mathrm{p}-$ Si structure in planar geometry, J. Appl. Phys., 109(2011), 123924.

[2] K.Mok et al., Thickness independent magneto-optical coupling constant of nickel films in the visible spectral range, Rev. Sci. Instrum., 82(2011), 033112.

[3] K.Mok et al., Vector-magneto-optical generalized ellipsometry, J. Appl. Phys., 110(2011), 123110.

[4] K.Mok et al., Magneto-optical coupling in ferromagnetic thin films investigated by vectormagneto-optical generalized ellipsometry, Phys. Rev. B, 84(2011), 094413.

[5] G.Neuber et al., Temperature-dependent spectral generalized magneto-optical ellipsometry for ferromagnetic compounds, Rev. Sci. Instrum., 76(2005), 023910.

[6] Š.Višňovský, Magneto-optical ellipsometry, Czech. J. Phys. B 36 (1986), 625-650.

[7] R.M.A.Azzam, N.M. Bashara, Elipsometry and polirilized light, North Holland Publishing Company, New York, 1977.

[8] H.Fujiwara, Spectroscopic Ellipsometry Principles and Applications, Chichester, John Wiley \& Sons, 2007.

[9] O.A.Maximova et al., Magnetic contribution to Fresnel coefficients at magneto-ellipsometric investigation, Vestnik SibGAU, 49(2013), no. 3, 212-217.

[10] O.A.Maximova et al., Features of the ellipsometric investigation of magnetic nanostructures, Journal of Structural Chemistry, 55(1964), no. 6, 1134-1141. 
[11] O.A.Maximova et al., In situ magneto-optical ellipsometry data analysis for films growth control, JMMM, Available online 20 December 2016 (http://dx.doi.org/10.1016/j.jmmm. 2016.12.050).

[12] I.A.Tarasov et al., Quick ellipsometric technique for determining the thicknesses and optical constant profiles of $\mathrm{Fe} / \mathrm{SiO}_{2} / \mathrm{Si}(100)$ nanostructures during growth, Tech. Phys., 57(2012), no. $6,1225-1229$.

[13] J.A.Nelder, A.D.Mead, A Simplex Method for Function Minimization, Computer Journal, 7(1965), 308-313.

\section{Двухслойная модель отражающих ферромагнитных пленок для исследования тонких пленок методом магнитоэллипсометрии}

\section{Ольга А. Максимова \\ Сергей Г. Овчинников}

Институт физики им. Л.В. Киренского, КНЦ СО РАН

Академгородок, 50/38, Красноярск, 660036

Сибирский федеральный университет Свободный, 79, Красноярск, 660041

Россия

Николай Н. Косырев

Институт физики им. Л. В. Киренского, КНЦ СО РАН

Академгородок, 50/38, Красноярск, 660036

Россия

Сергей А.Лященко

Институт физики им. Л. В. Киренского, КНЦ СО РАН

Академгородок, 50/38, Красноярск, 660036

СибГАУ им. Академика М. Ф. Решетнева Красноярский рабочий, 31, Красноярск, 660037

Россия

Представлен метод анализа магнито-эллипсометрических измерений. Детально рассматривается двуслойная модель ферромагнитных отражающих пленок. Полученный алгоритм может использоваться для контроля оптических и магнито-оптических свойств пленок в процессе их роста в вакуумных камерах.

Ключевые слова: магнито-оптическая эллипсометрия, эффект Керра, двухслойная модель, ферромагнетик, отражение, контроль роста. 